infants recover when dietary chloride supplements are provided. Previous follow-up reports have drawn attention to developmental delay (Chutorian et al. Pediatr Neurol 1985; 1:334-341) and the present study demonstrates specific cognitive, language and attentional deficits in later childhood.

\title{
MAPLE SYRUP URINE DISEASE AND CEREBRAL EDEMA
}

Cerebral edema causing death in four children with maple syrup urine disease (MSUD) is reported from St. Christopher's Hospital for Children, Temple University School of Medicine, Philadelphia, PA. An intercurrent infection that caused severe dehydration and acidosis precipitated the cerebral edema when the children were three to five years of age. All four had been adequately managed with few problems before the intercurrent illness. The diagnosis of cerebral edema was established by autopsy in one patient and demonstrated by CT in two, one also having a subarachnoid hemorrhage. The authors recommend early hospitalization and cautious rehydration in all children with MSUD in whom intercurrent infections develop in association with decreased nutrient intake or vomiting, or both. Early treatment of dehydration and acidosis may prevent the catastrophic consequences observed (Riviello JJ Jr et al. Cerebral edema causing death in children with maple syrup urine disease. J Pediatr July 1991; 119:42-45).

COMMENT. Acute metabolic decompensation in MSUD during otherwise minor illnesses has generally been presumed to result from massive release of leucine from protein catabolism. The dynamics of protein metabolism and implications for management are reported from the Murdoch Institute and the Department of Dietetics, Royal Children's Hospital, Melbourne, Australia, and the Nutrition Research Group, Clinical Research Centre, Harrow, UK (Thompson GN et al. $\underline{J}$

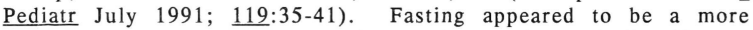
important cause of increased leucine levels than the catabolic effect of infection. Branched-chain amino acid restriction should be commenced at the start $n$ minor illness in children with MSUD and the intake of other nutrients should be increased. Dietary supplementation reduces the risk of metabolic decompensation during acute illnesses and early and meticulous treatment of MSUD results in intellectually normal children according to a study from the Division of Biochemical Development and Molecular Diseases, Children's Hospital of Philadelphia, University School of Medicine, Philadelphia (Kaplan P et al. J Pediatr July 1991; $\underline{119}: 46-50)$. Affected children treated presymptompatically had higher IQ scores than their siblings treated when their disease was symptomatic.

\section{NON-MENKES TYPE COPPER DEFICIENCY}

A two year old girl with copper deficiency progressive neuronal disorder and granulocytopenia is reported from the Department of Pediatrics, Kyoto University, Japan. A familial granulocytopenia was noted at one month 